\begin{tabular}{|c|c|}
\hline \multirow{3}{*}{ 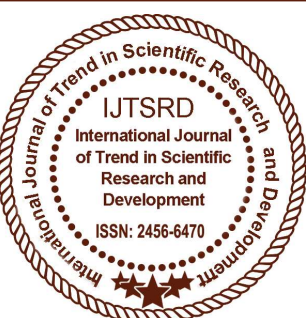 } & $\begin{array}{l}\text { International Journal of Trend in Scientific } \\
\text { Research and Development (IJTSRD) }\end{array}$ \\
\hline & International Open Access Journal \\
\hline & ISSN No: 2456 - 6470 | www.ijtsrd.com | Volume - 2 | Issue -3 \\
\hline
\end{tabular}

\title{
Operating System a Case Study
}

\section{Sumanta Kabiraj}

B.Sc. in Computer Science, Kazi Nazrul University, T.D.B College Raniganj, Raniganj, West Bengal

\author{
Prof. Subhashis Kumar Chandra \\ Kazi Nazrul University, T.D.B \\ College Ranuganj, Raniganj, \\ West Bengal
}

\begin{abstract}
Anjli Gupta
B.Sc. in Computer Science, Kazi Nazrul University, T.D.B College Raniganj, Raniganj, West Bengal
\end{abstract}

\section{ABSTRACT}

An Operating System or OS is the most important software and program that can run all types of Computer, Mobile phone, Hand Held Device, etc. It helps to connect and interface computer hardware to program and application software and other common essential services. The OS is initially loaded on at boot time. Like a host, the OS acts for running and handling of application programs and operation of the hardware on the machine. The working principle of OS is first to configure then check ability to perform and then allow the program to run. Its main purpose is to handle the activities based on computer hardware. The OS also provides management of input, output and storage devices. Mostly in a system, some different computer programs run at the same time and they need to access CPU, memory, and storage. In an operating system, the user can communicate with the Command Line Interface by typing some command, and the user receives the response back from the system. But Modern Time Operating system uses a graphical user interface or GUI for easy to use. Now user can communicate with a system using a mouse to click button, menus, and everything is displayed on the screen using Text, Graphics or image. It is like a bank manager-- it makes sure that the working of application software must not be interfered by any other activity.

Keywords: Introduction, Type of operating system, operating system service, security, function of operating system, Example of some operating system

\section{INTRODUCTION}

A Computer Machine or System has the different types of device and software which are used to do a complete operation or task. In Computer Operating System ${ }^{[1]}$, the commonly used and important resource is Computer Memory(RAM, ROM), Storage Device(HDD, FDD, SDD), CPU(Central Processing Unit), Processors and Other Input / Output Device. ${ }^{[6]}$ The computer Operating System manage and operate the whole computer system and above resource element and allocate them to perform a specific task or operation or program. In short terms, we can say that the operating system is an interface that connects the user to the system and other application software to hardware. Without an operating system, the general purpose computer is useless ${ }^{[2]}$.

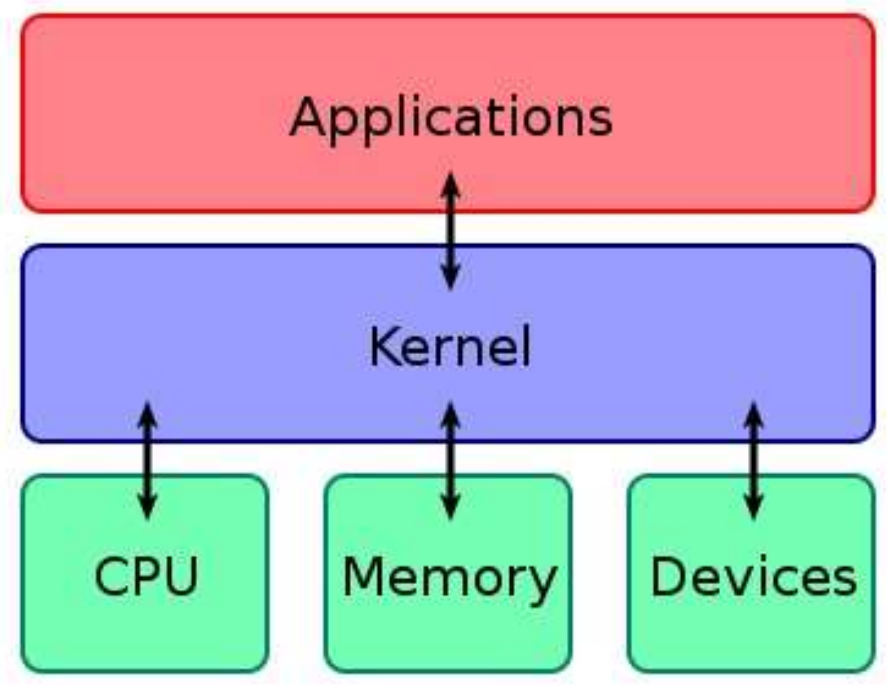

The operating system is designed to communicate with the user, computer system, and application software. 
For the easy operation of the operating system, we can see two types of view of every operating system.

That is---

1. User View ${ }^{[2]}$ - From the user viewpoint, the operating system was designed to maximize the performance of work and provide the minimal effort of the user for any operation. Such system is designed to monetize the individual user resource. In this case, the operating system is designed for most uses, some attention is given to performance, and no payment is made for the use of the resource.

2. System View ${ }^{[2]}$ - From the system viewpoint, the operating system is a hardware-based program. Operating System assigns the allocated memory for the different process of every resource. It shares the control resources among the programs. And also control the incorrect usage, error and deadlock condition. This is a program that always runs on the system in the form of the kernel. It controls the application program that is not part of the kernel.

\section{Types of Operating System}

The operating system array is being developed from the first computer generation and with the tip period ${ }^{[3]}$

1) Simple Batch Operating System: In this types of system there is no direct connection between computer and computer user. ${ }^{[3]}$ user push a operating data with punch type card or magnetic tape, after submitting to the operation system, operator batch together with their requirement then the system manage each program in batch and execute the operation.

2) Multiprogramming Batch System: In this types of operating system, system picks the jobs from his memory, when the job need an input and output operation the OS switch another job to another job. If the many job run on same time the OS work on a method called Scheduling.

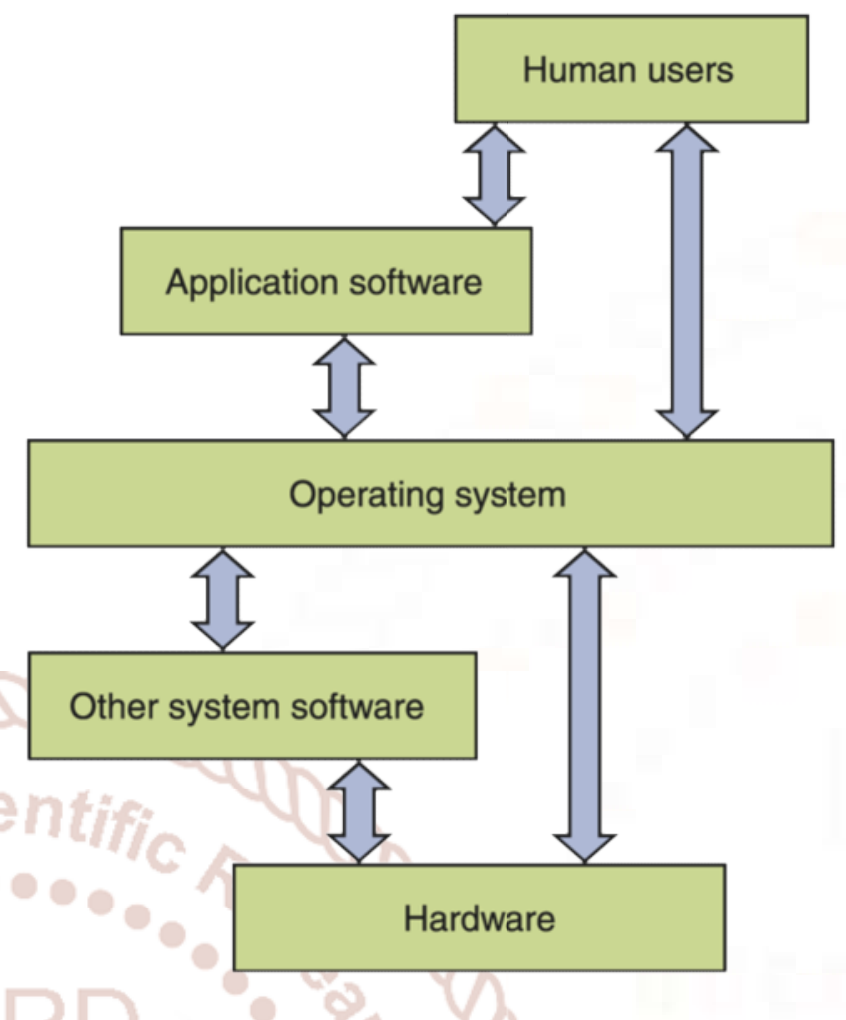

3) Single User, Single Task Operating System: In this type of system, computer works on single task on a single user at a time. The Palm OS is the good example for this type of computer.

4) Single User, Multi Task Operating System: This system allows to work with one or more than one process at same time.

5) Multi User Operating System: In these type of OS, system permit multiple user to access the same data or information at the same time via network or other connection method. ${ }^{[4]}$ the user can interact with each other. Some example these is Windows 7, Linux and Unix.

6) Multiprocessor System: Multiprocessor system consists many several processor to complete a process. In this types of system OS provide higher power and speed. The all process or operation in a Multiprocessor System operate the under single OS. The best feature of this type of operating system is the performance was enhanced, increase the system throughput, Speedup the execute of a single process.

7) Desktop System: This type of OS specially build for Desktop or Personal Computer/ Mainframe Computer. the goals of these operating system maximize user experience, convenience and responsiveness. Windows and Mas OS was a Desktop OS. ${ }^{[5]}$ 
8) Distributed Operating System: The Distributed Operating System use to multiple processor to serving multiple application and user. The processor connect or communicate with each other through different communication method such as bus, wired connection, telephone lines etc. The processor of distributed system are referred as sites, node, port etc.

9) Network Operating System: The network Operating System runs on server and provides the user to manage data, security, application and other network related function. The website was hosted in Network Operating System. The main uses of this type of operating system is shared file, printer and other device among multiple computer on this network with connected via LAN, PAN, WAN or other network connected method.

10) Time Sharing Operating System: In this types of operating system works on more than one process or task one by one at a time. the processor divide the time was distributed among the all executed process or task. The processor switch rapidly between the process.

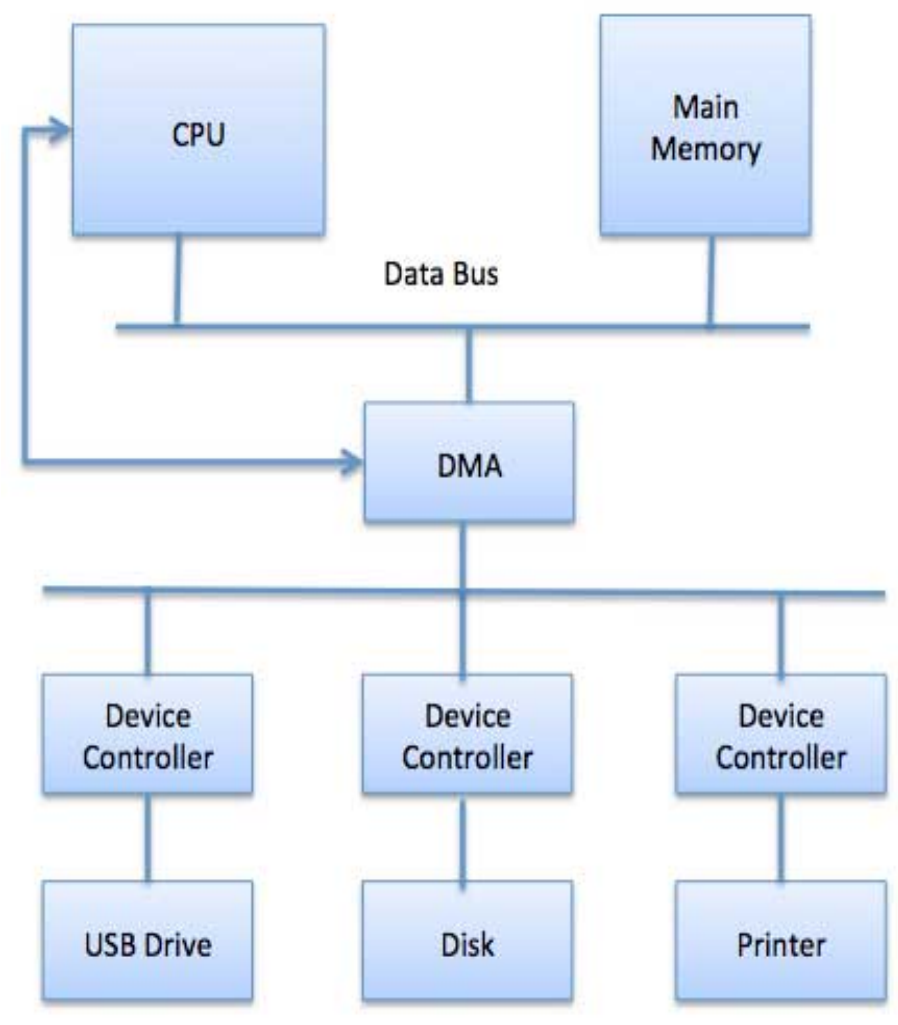

11) Real-time Operating Systems: A real-time system is defined as a data processing system, the time difference for input processing and feedback is so small that it controls the environment. The system displays the time-consuming information and is marked as response time. So in this way, the reaction time compared to online processing is very low.

12) Hand-held System: This time of OS specially made for Mobile and Tablet Like Hand Held Device. The hand-held devices have Personal Digital Assistance with network connectivity. Hand-held device have a limited memory and Small Power Supply, for this reason the OS is memory and power efficient. The OS not used virtual memory. Some hand-held have some wireless connectivity like Bluetooth, WiFi to access Internet and also media process like play music and camera for expend their utility.

\section{Operating System Service:}

Operating System Provide a Service for User and System for an environment to execute the program and the programs in a convenient manner.

1) Program Execution: OS handle different types of activities or Program in own system.

\section{System Program Like:}

a) Name Server,

b) File Server,

c) Spooler,

d) Printing Process,

e) Run a Specific Program

f) Registers,

g) Code to Execute

h) Data Manipulate.

\section{Main Program Management Activities -}

a) Execute Program.

b) Deadlock Handling.

c) Loads Program into Memory.

2) Input-Output Operation: Operating System Manage the connection between user, device and device driver.

Each I/O device was operated with their Device Driver and Communicate with OS. 
a) OS provides access to required $\mathrm{I} / \mathrm{O}$ device.

b) Permission for reading or Write operation on any file by I/O Device.

3) File System Manipulation: File Means the Data, the collection of information that stored on disk either secondary or primary, long time or short time uses purpose. Storage Media like Magnetic Disk, Magnetic Tape, Solid State Disk, and Optical Drive.

For each media that store in Storage device having its own properties like -

a) Transfer Rate,

b) Storage Capacity,

c) Transfer Speed,

d) Data access Permission (Read Only, Editable, and Hidden)

e) Data Access Method

In an Operating System, File was normally stored or organized in the directory or a folder for easy navigation and uses and find the reach of file.

In a Directory contain File or another directory.

Some main File Management Activities -

a) Read or write a file.

b) Permission for operation or uses of the file.

c) OS Provides Interface for Create, Edit or Update, Delete Files and Folders or Directories.

d) Its Provide Interface for Backup and Restore the File System.

4) Communication: Some Main activities of OS with respect to communication -

a) Two processes required to transfer data between them.

b) The Communication process, one computer or one

Computer to another computer by a computer network

c) Communication process was accomplished by two methods, by shared memory or Message Passing.

5) Error Detection: In an operating System Error occurs anytime and anywhere-
1) Simple Batch Operating System : In this types of system there have no direct connection between computer and computer user. ${ }^{[3]}$ user push a operating with punch type card or magnetic tape, after submit the operation system operator batch together with their requirement then the system manage each program in batch and execute the operation.

2) Multiprogramming Batch System : In this types of operating system, system picks the jobs from his memory, when the job need an input and output operation the OS switch another job to another job. If the many job run on same time the OS work on a method called Scheduling.

3) Single User, Single Tusk Operating System : This type computer works on single task on a single user at a time. The Palm OS is the good example for this type of computer.

4) Single User, Multi Task Operating System : This system allows to work with one or more than one process at same time.

5) Multi User Operating System : In these type of OS, system permit multiple user to access the same data or information at the same time via network or other connection method. ${ }^{[4]}$ the user can interact with each other. Some example these is Windows 7, Linux and Unix.

6) Multiprocessor System : Multiprocessor system consists many several processor to complete a process. In this types of system OS provide higher power and speed. The all process or operation in a Multiprocessor System operate the under single OS. The best feature of this type of operating system is the performance was enhanced, increase the system throughput, Speedup the execute of a single process.

7) Desktop System : This type of OS specially build for Desktop or Personal Computer/ Mainframe Computer. the goals of these operating system maximize user experience, convenience and responsiveness. Windows and Mac OS was a Desktop OS. ${ }^{[5]}$

8) Distributed Operating System : The Distributed Operating System use to multiple processor to serving multiple application and user. The processor connect or communicate with each other through different communication method such as bus, wired connection, telephone lines etc. The processor of distributed system are referred as sites, node, port etc. 
9) Network Operating System : The network Operating System manage on server and must be provide by the user to manage the data, security, application and other network related task. The website was hosted in Network Operating System. The main uses of this type of operating system is shared file, printer and other device among multiple computer on this network with connected via LAN, PAN, WAN or other network connected method.

10) Time Sharing Operating System : In this types of operating system system works on more than one process or task one by one at a time. the processor divide the time was distributed among the all executed process or task. The processor switch rapidly between the process.

11) Real-time Operating System : A real-time system is defined as a data processing system, the time difference for input processing and feedback is so small that it controls the environment. The system displays the time-consuming information and is marked as response time. So in this way, the reaction time compared to online processing is very low.

12) Hand-held System : This time of OS specially made for Mobile and Tablet Like Hand Held Device. The hand-held device have Personal Digital Assistance with network connectivity. Hand-held device have a limited memory and Small Power Supply, for this reason the OS is memory and power efficient. The OS not used virtual memory. Some hand-held have some wireless connectivity like Bluetooth, WiFi to access Internet and also media process like play music and camera for expend their utility.

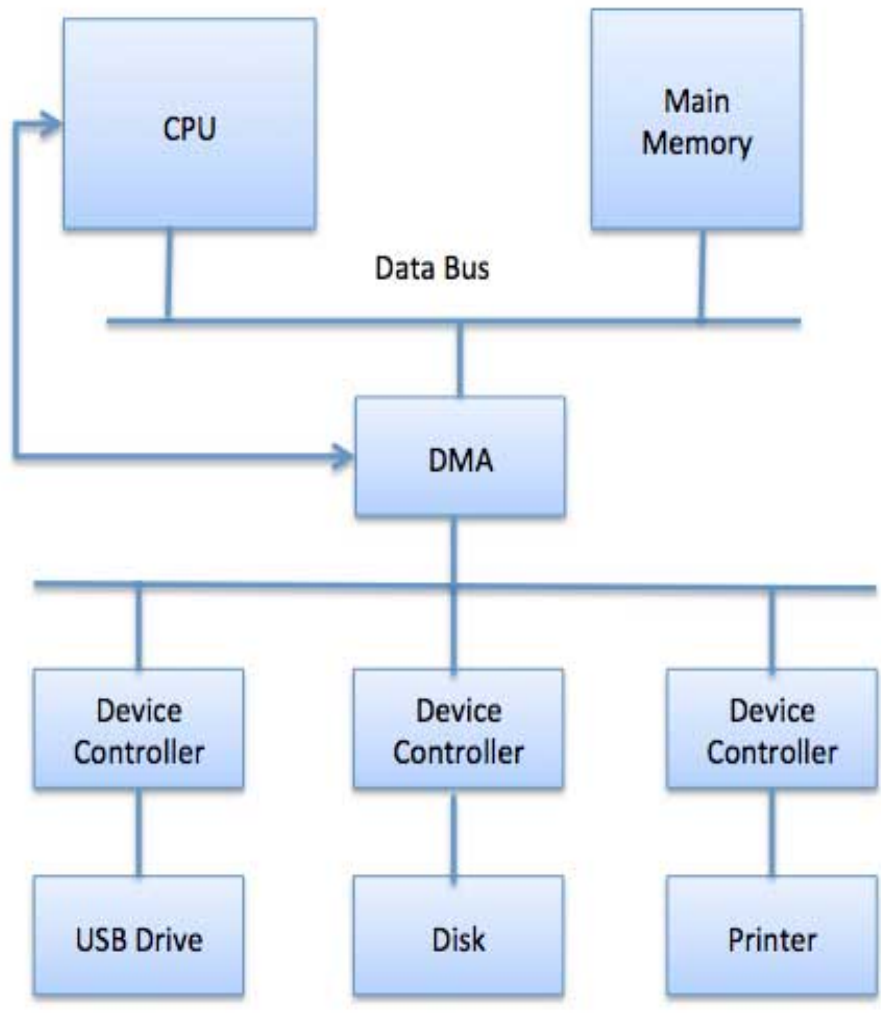

III. Operating System Service:

Operating System Provide a Service for User and System for an environment to execute the program and the programs in a convenient manner.

1) Program Execution: OS handle Different types of Activities or Program in Own System.

\section{System Program Like:}

a) Name Server,

b) File Server,

c) Spooler,

d) Printing Process,

e) Run a Specific Program

f) Registers,

g) Code to Execute

h) Data Manipulate.

Main Program Management Activities -

a) Execute Program.

b) Deadlock Handling.

c) Loads Program into Memory. 
2) Input-Output Operation: Operating System Manage the connection between user, Device and Device Driver.

Each I/O device was operated with their Device Driver and Communicate with OS.

a) OS provides access to required $\mathrm{I} / \mathrm{O}$ device.

b) Permission for reading or Write operation on any file by I/O Device.

3) File System Manipulation: File Means the Data, the collection of information that stored on disk either secondary or primary, long time or short time uses purpose. Storage Media like Magnetic Disk, Magnetic Tape, Solid State Disk, and Optical Drive.

For each media that store in Storage device having its own properties like -

a) Transfer Rate,

b) Storage Capacity,

c) Transfer Speed,

d) Data access Permission (Read Only, Editable, and Hidden)

e) Data Access Method

In an Operating System, File was normally stored or organized in the directory or a folder for easy navigation and uses and find the reach of file.

In a Directory contain File or another directory.

Some main File Management Activities -

a) Read or write a file.

b) Permission for operation or uses of the file.

c) OS Provides Interface for Create, Edit or Update, Delete Files and Folders or Directories.

d) Its Provide Interface for Backup and Restore the File System.

4) Communication: Some Main activities of OS with respect to communication -

a) Two processes required to transfer data between them.

b) The Communication process, one computer or one
Computer to another computer by a computer network

c) Communication process was accomplished by two methods, by shared memory or Message Passing.

5) Error Detection: In an operating System Error occurs anytime and anywhere. The error also may occur in CPU or Input Output Device or Memory and Hardware and OS handled all the Errors.

Some Major Operating System with Respect to Error -

a) The Operating System checks all the Error Constantly.

b) The OS handled and take an appropriate action to detect and resolve all the errors.

6) Resource Allocation: Resource like main memory, $\mathrm{I} / \mathrm{O}$ device, CPU, and Storage. All the resource management by the OS and file Storage are allocated for each and different job.

Some main Resource Management -

a) The OS scheduled all kind of Resource using Schedulers.

b) All CPU Schedulers algorithms used for better CPU Performance and Utilization.

7) Protection: Consider a computer System has multiple users with multiple processors, for that reason, the various processes should be protected from each other user, process, and Activities.

Protection is method or mechanism or way to driving the access of every process, program, users or resource that makes a computer system.

Some main Activities of an operating system -

a) The OS protect Input Output Device from External invalid Access.

b) The OS authenticate the user by password or different authentication method.

Storing any Data or Information in two methods -

1) Contiguous Storage Allocation: This is the simplest Storage allocation technique. In this type of allocation technique the data or any program was allocate contiguously. Operating System was allocates the estimate amount of memory required by complete the process before allocation. 
2) Non - Contiguous Storage Allocation: The name indicates the program and related data should not be saved in the adjacent location; the program is distributed in a different location. When a process requires an access to the component, the OS provides access to this assignment table when a table puts a record.

In real life the primary memory was not sufficient for any operation to store the whole program. In this reason OS take help of Virtual Storage technique.

There are two approaches to Virtual Allocation Storage---

1) Program Paging - A program is broken in fixed size of page and stored in secondary memory. The page is given by the OS as logical or virtual address from 0 to $\mathrm{n}$. A method called Page table is used to lead the maps from logical to physical address which is used to retrieve the page when required.

2) Program Segment - A program is divided into small logical segment, assigned logical address from 0 to $\mathrm{n}$ and stored in secondary memory. A method call segment table is used to lead segment from secondary memory to primary memory.

Operating System are used both of Virtual Storage allocation to store on computer in form of files to optimize the memory uses. A large program part by different segment into page or more the one segment may be stored in a single page.

4) File Management: File is mainstream of a computer. Data or information stored in computer in a file. The user keeps their data safely and correctly in a file that's important for and OS. The management of file system and work with file is known as or called as File Management. ${ }^{[17]}$

The File management required some tools or operation to work with file.

1) Create - Create a New file for storing data or Information.

2) Update - Update some information of old created file.

3) Read - Open and read the data of a created file.

4) Delete - Delete the created File.

5) Recover - Recover the Deleted or corrupted File.
6) Sharing - Sharing the created file to Different System.

7) Secure - Secure the data of file with the use of Password and Encryption.

5) Memory Management: Computer memory optimization, memory control and overall system

Performance is known as Memory Management. In modern computer the memory management plays an important role in operating System ${ }^{[13]}$.

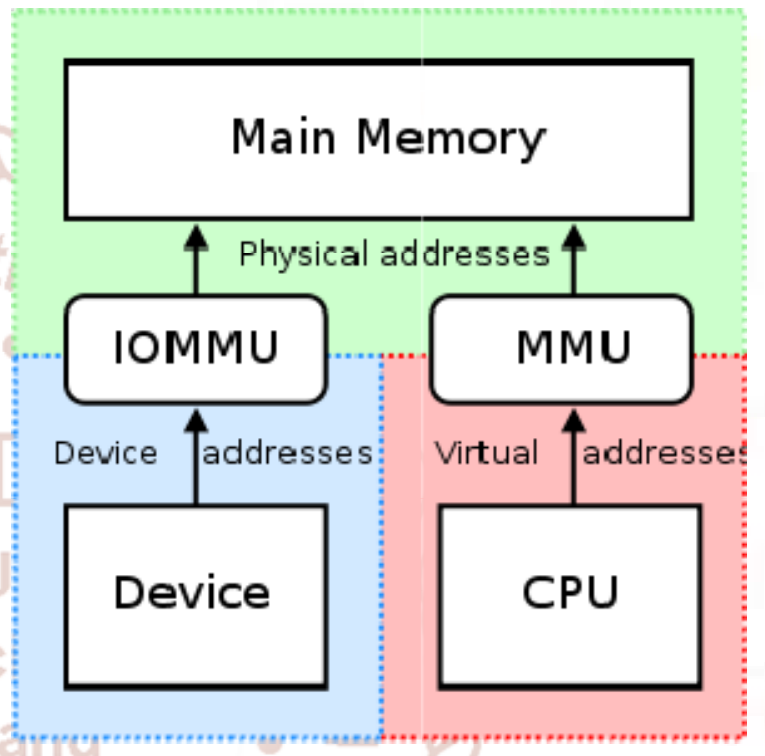

The computer systems have two types of memory -

1) Primary Memory - Primary Memory is a fast or direct memory. It's also expensive memory. Primary Memory is used to run the executed programs and service and stored in a specific memory location temporary. It's also be known as Main Memory. RAM, ROM, and Cache Memory were Primary Memory.

2) Secondary Memory - Secondary memory is less expensive but slower memory as Primary Memory. The user store data and information stored in secondary memory. Common secondary memory was Hard Disk Drive, Solid State Drive, Optical Disk, Pen Drive etc.

To maintain a balance between the OS to ensure that the performance of the system is not damaged due to very early primary memory or system expenditure.

Need to maintain intermediate access and recovery functionality for high system performance input and output data, user instructions and performance information. Once program requests are accepted, as 
per the requirement, it allocates primary and secondary storage areas. Once the infection is completed, the allocated memory space is free. Using a storage management strategy to keep all storage space allocated or free the allocation.

6) Device Management: The operating system interacts with the peripheral device with specific device driver software for peripheral control. In a Computer the device management system is a very small embedded system and device management routines may be included in Operating System.

When many process in operating system access the device or request for access to the device, the operating system manage all device in a specific way that partially shares that device and operate the among all process. Process Access device operate by a method call System Call Interface ad its interface provide by the OS.

\section{Examples of Some Operating System:}

The some common operating was found for personal computer. The three most used and most common Operating System was build for Personal User and Personal Computer.

The Operating System is-

1) Microsoft Windows: The most useable personal Computer and Home Computer Operating System ${ }^{[21]}$. It was made and Market placed by Windows. The Operating System was fully closed source. It's a GUI Operating System. In Windows includes various types of greater functionality like native File Manager, Program Manager. It has dynamic interface. Windows offered including network windows for work with

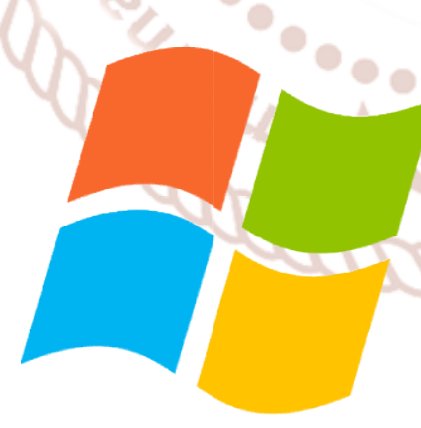
workgroup for aimed to business.

Windows also provide network server for hosting, many website and web server was run with windows. Approx $90 \%{ }^{[23]}$ Computer run in windows.

Some Famous Windows Operating System ${ }^{[7]}$ Versions are

a) Windows 10

b) Windows 8.1 or 8 c) Windows Home Server

d) Windows Server 2008

e) Windows 7

f) Windows Vista

g) Windows XP

h) Windows 2000

i) Windows NT

j) Windows ME (Windows Millennium Edition)

k) Windows 98

1) Windows 95

m) Windows $3.0-3.1$

n) Windows $1.0-2.0$

o) Ms-Dos

2) Mac OS: Mac OS (Macintosh Operating System) is a Graphical Operating System ${ }^{[15]^{\text {it }}}$ was developed by Apple Inc. It's a

Unix and Family

Operating System.

And it's the primary or main operating system 7 of Apple's Family. It's used on

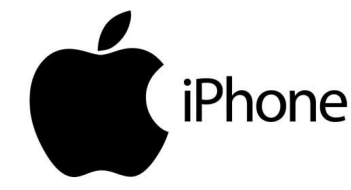

Stuck on Apple Logo Laptop, Desktop and home computer.

Some Famous Mac OS versions are ${ }^{[8]}$.

a) Version 10.13: "High Sierra"

b) Version 10.12: "Sierra"

c) Version 10.11: "El Capitan"

d) Version 10.10: "Yosemite"

e) Version 10.9: "Mavericks"

f) Version 10.8: "Mountain Lion"

g) Version 10.7: "Lion"

h) Version 10.6: "Snow Leopard"

i) Version 10.5: "Leopard" 
International Journal of Trend in Scientific Research and Development (IJTSRD) ISSN: 2456-6470

j) Version 10.4: "Tiger"

k) Version 10.3: "Panther"

1) Version 10.2: "Jaguar"

m) Version 10.1: "Puma"

n) Version 10.0: "Cheetah"

o) Public Beta: "Kodiak"

3) Linux: Its Provide By Linux Distribution. Linux is a Open Source Operating System, ${ }^{[9]}$ Open Source Operating System means anyone can be modified, change anything ${ }^{[10]}$, Distributed by any one in whole world and can use it free.
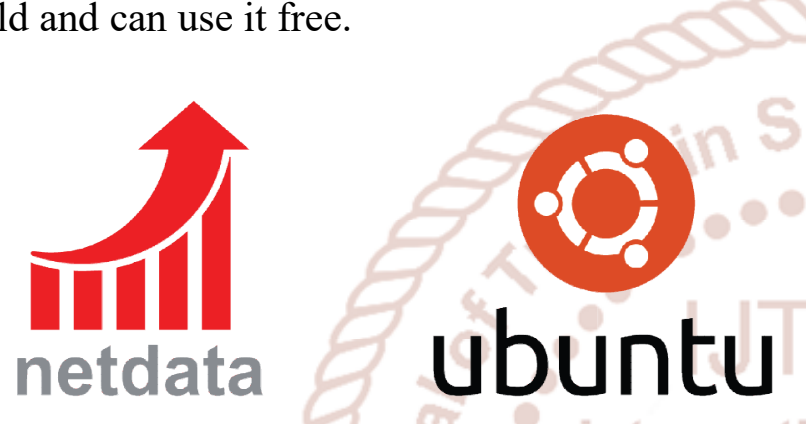

Some Linus OS for Web Servers -
a) Ubuntu Server
b) Red Hat Enterprise Linux
c) CentOS
d) SUSE Enterprise Linux`

All this operating System was made first time in CLI (Coding Line Interface) ${ }^{[25]}$ Platform and modern Operating System use GUI (Graphical User Interface) ${ }^{[24]}$.

The Some Operating System that found On Mobile or Handheld Device ${ }^{[27]}$.

1) Android: Android is a Famous Mobile Operating System that is developed by Google. The Android OS work on Mobile, Wear Device, Television, Projector, Tablet, and Car Variant Types of Device. This

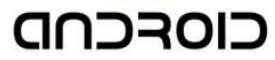
android is an open

Its different from Proprietary Software like $n$ source software and its build on Linux Kerne ${ }^{[14]}$. Windows(Owner - Microsoft) and Mac (Owner Apple), which can only modified, distributed by only the own company. The advantages of Linux is its totally free, many distributors, found different versions that anyone choose from it.

The Most Web Server and Website are Running on Linux OS because it's relatively easy to edit.

Some Well-Known Linux Distribution Operating

System for Personal Computer -

Today almost 70\% Mobile uses Android.

2) iOS: iOS is also known as iPhone OS. The iOS mobile operating system was created and developed by Apple Inc. exclusively for his own device Hardware. The operating System also runs on iPad, Apple Watch and Also some Apple Device. ${ }^{[20]}$

3) BlackBerry OS: BlackBerry OS is a mobile Operating System developed by BlackBerry Limited for its own BlackBerry Linear Smart-phone or Other BlackBerry Hand-held Device ${ }^{[18]}$.
a) Ubuntu
b) Kali-Linux
c) Linux Mint
d) Fedora
e) Debian
f) OpenSUSE
g) Deepin
h) Red-Hat

4) Symbian: Symbian was a mobile operating System that's specially designed for Smart-phone and it's also known as Nokia Symbian OS. Itis a close source Operating System.

5) WebOS: webOS is also built in Linux karnel;it's a Multitasking Operating System. The webOS was developed by Palm Inc and next it's acquired by HP. Now it is an Open Source Operating System. The Operating system was run on Smart phone and smart $\mathrm{TV}^{[19]}$.

6) Windows OS: It is developed by Microsoft Corporation and runs on Computer. Specially made an 
Operating System for Mobile that run on Microsoft Mobile.

7) KaiOS: Itisan Open source operating system from Firefox Organization. It is a web based operating system. It is made for smart-phone with the affordable features of phone. Today KaiOS support 4G service. KaiOS was run on Reliance Jio Provide Jio-Phone.

\section{CONCLUSION}

In the same way, the computer OS controls your desktop, a mobile operating system is a software program that control your mobile phones, tablets, ipads. Some well known operating systems are Android, Windows, Black berry, and iOS. These all runs on mobiles or tablets. The mobile $\mathrm{OS}$ is responsible for determining the functions and features available on your device such as keyboards, thumb wheel, synchronize with applications such as email, text messaging and more. It will also determine that which extra mobile apps can be used on your device. So, we can easily conclude that operating system plays an important role in our everyday lives. We can also say that a computer without an operating system is just like a human body without heart. Therefore, operating system is an essential part of our living system.

\section{REFERENCES:}

1. Introduction http://openbookproject.net/courses/intro2ict/syste $\mathrm{m} / \mathrm{os}$ _intro.html

2. https://www.studytonight.com/operatingsystem/introduction-operating-systems

3. https://en.wikipedia.org/wiki/Operating_system

4. https://www.tutorialspoint.com/operating_system/ os_types.htm

5. https://www.studytonight.com/operatingsystem/types-of-os

6. https://www.gcflearnfree.org/computerbasics/unde rstanding-operating-systems/1/

7. Windows Version https://en.wikipedia.org/wiki/Microsoft_Windows

8. Mac OS X Version https://en.wikipedia.org/wiki/MacOS_version_hist ory

9. Windows VS Linux http://windowsvslinux.wikidot.com/
10. http://www.renewablepcs.com/aboutlinux/advanta ges-of-using-linux

11. Security http://windowsvslinux.wikidot.com/\#toc4

12. https://www.tutorialspoint.com/operating_system/ os_security.htm

13. Memory Management https://en.wikipedia.org/wiki/Memory_manageme $\mathrm{nt}$

14. Android OS Wiki https://en.wikipedia.org/wiki/Android_(operating _ system)

15. Mac OS X https://en.wikipedia.org/wiki/MacOS

16. Function Of Operating https://www.tutorialspoint.com/operating_system/ os_overview.htm

17. File Management http://www.includehelp.com/operatingsystems/file-management-functions.aspx

18. BlackBerry OS https://www.techopedia.com/definition/25196/bla ckberry-os

19. WebOs http://whatis.techtarget.com/definition/webOS

20. iOS https://www.lifewire.com/what-is-ios1994355

21. Windows OS https://en.wikipedia.org/wiki/Microsoft_Windows

22. Dhotre, I.A. (2009). Operating Systems. Technical Publications.

23. OS Statistics

https://www.w3schools.com/browsers/browsers_o s.asp

24. GUI Os

https://www.webdesignerdepot.com/2009/03/oper ating-system-interface-design-between-19812009/

25. CLI OS

http://searchwindowsserver.techtarget.com/definiti on/command-line-interface-CLI

26. GUI vs CLI

https://www.cybrary.it/0p3n/command-lineinterface-cli-vs-graphical-user-interface-gui/ $\wedge$

27. Handheld Device http://searchmobilecomputing.techtarget.com/defi nition/handheld 